\title{
Educación sexual y perfil del adolescente de nivel secundario en San Juan de Lurigancho - Lima
}

\author{
Alejandra Francesca Parodi-Carro ${ }^{1}$
}

Parodi-Carro AF. Educación sexual y perfil del adolescente de nivel secundario en San Juan de Lurigancho - Lima. Cuid salud, jul-dic 2014; 1(2).

\section{RESUMEN}

Introducción: en América Latina y el Caribe, el $22 \%$ de adolescentes entre 15-19 años es sexualmente activo. Uno de cada cinco embarazos ocurre entre los 12-19 años, principalmente en el distrito de San Juan de Lurigancho (SJL). Objetivo: determinar el nivel de conocimiento sobre educación sexual y su perfil en una población de adolescentes de secundaria en el distrito SJL. Metodología: enfoque cuantitativo, método observacional y diseño descriptivo, realizado en SJL-Lima en escuelas de secundaria, tomando una población de 1200 adolescentes entre 13-17 años y una muestra probabilística de 449 . Los datos se recolectaron mediante cuestionario entre abril-junio/2014 y analizados con estadística descriptiva. Resultados: el $61 \%$ de adolescentes presentó un nivel medio de conocimiento sobre educación sexual, destacándose las dimensiones: sexualidad, métodos anticonceptivos y ética. Contrariamente, existen vacíos sobre la eficacia de métodos anticonceptivos y uso correcto del preservativo, minimizando la importancia de los valores en la relación amical/pareja. En el perfil sexual, el $12 \%$ se inició sexualmente a los 13 años, siendo que el $12 \%$ tenía perfil de riesgo. Conclusiones: el conocimiento sobre educación sexual en adolescentes fue de nivel medio, con un perfil sexual protector. Siendo necesario seguir trabajando en esta problemática y recuperar la práctica valores, promoviendo la abstinencia sexual.

Palabras clave: adolescencia, sexualidad, educación sexual, embarazo precoz, enfermería.
Parodi-Carro AF. Sexual education and Adolescent profile in high school from San Juan Lurigancho Lima. Cuid salud, jul-dic 2014; 1(2).

\section{ABSTRACT}

Introduction: In Latin America and the Caribbean, 22\% of adolescents among 15-19 years aged are sexually active. In Peru, one of every five pregnancies occurs among 12-19 years, mainly in the district of San Juan de Lurigancho (SJL). Objective: To determine the level of knowledge about sexual education and its profile in a population of adolescents at high school in the district SJL. Methodology: Quantitative approach, method observational and descriptive design, made in SJL-Lima in high schools, taking a population of 1200 adolescents among13-17 years and probabilistic sample $(n=449)$. The data was collected through a questionnaire among AprilJune/2014 and analyzed using descriptive statistics. Results: $61 \%$ of adolescents presented a middle level of knowledge about sexual education, highlighting the dimensions: sexuality, contraception, and ethics. In contrast, there are empty on the effectiveness of contraceptive methods and proper condom use, minimizing the practice of values in the relationship friendly/couple. In the sexual profile, $12 \%$ were sexually initiated at the age of 13 , although the majority has a protective profile and $12 \%$ of risk. Conclusions: Knowledge on sexual education in adolescents was middle level, with a sexual profile protector. It still needs to continue working on this issue and recover the practice of values, promoting sexual abstinence.

Keywords: Adolescence, sexuality, sex education, early pregnancy, nursing.

\footnotetext{
${ }^{1}$ Licenciada en Enfermería. Egresada de la Escuela de Enfermería Padre Luis Tezza, afiliada a la Universidad Ricardo Palma, LimaPerú.
} 


\section{INTRODUCCIÓN}

La adolescencia es considerada una etapa compleja en la vida del ser humano, en la que existe necesidad de búsqueda de nuevas experiencias y sensaciones, entre ellas las relaciones sexuales con los pares, exponiéndose a diversos factores de riesgo como el embarazo precoz, ${ }^{1}$ además de las infecciones de transmisión sexual (ITS).

Actualmente, existe un incremento en las tasas de natalidad adolescente, lo que evidencia la cantidad de embarazos precoces de los últimos años, situación que constituye un problema social, económico, laboral y sanitario. ${ }^{2}$

Encuestas aplicadas en diversos países, muestran que el $11 \%$ de mujeres y $6 \%$ de varones entre 15-19 años, aseguran haber tenido relaciones sexuales antes de los 15 años, siendo esta proporción mayor en América Latina y el Caribe $(22 \%) ;{ }^{1}$ con embarazos cada vez más precoces, destacando que una de cada cuatro personas está en la adolescencia, a diferencia de los países desarrollados que tienen una de cada siete. ${ }^{3}$

El embarazo adolescente afecta a todos los estratos sociales, con predominio en el nivel socioeconómico bajo. ${ }^{4} \mathrm{Al}$ que se puede adicionar las elevadas tasas de ITS, factor de vulnerabilidad en los adolescentes y elevada probabilidad de adquirir el virus de inmuno-deficiencia humana (VIH). En algunas regiones del mundo, casi la mitad de los casos de infección por VIH ocurre en menores de 24 años; siendo las mujeres las más afectadas en comparación con los varones, estimando que por cada dos mujeres que no usan métodos anticonceptivos de ninguna clase, un varón utiliza preservativo. ${ }^{5}$ El Ministerio de Salud, ${ }^{6}$ citando la Encuesta Demográfica y de Salud Familiar (ENDES) 2012, revela que las adolescentes sexualmente activas y unidas a su pareja son las que menos usan preservativos en comparación con adolescentes no unidas a su pareja. Por consiguiente, el embarazo es un evento esperado y frecuente al inicio de la vida sexual activa, incrementando los riesgos derivados de la maternidad precoz, pues entre más joven se presente el embarazo, menos capacidad tendrá la adolescente de criar un niño sano y, aún más, desarrollarse como mujer profesional trabajadora en potencia. ${ }^{7}$

Según el INEI 2012, ${ }^{8}$ la mayor cantidad de adolescentes que inician la experiencia sexual lo hacen por decisión personal, falta de planificación y de comunicación; en general, las mujeres omiten su voluntad propia y acceden a las relaciones sexuales por complacer a su pareja; al contrario del varón, quien lo hace para tener experiencia. Destacando que en esta etapa la inmadurez emocional y física hace que confundan amor o cariño de pareja por el simple "deseo".

Debe recordarse que en el país el 2006, existía una ley que penalizaba las relaciones sexuales entre menores de edad y de mayores a menores, la cual fue abolida en julio del $2008 ;^{9}$ observándose luego incremento en las tasas y porcentajes de embarazo adolescente, así como también la muerte en adolescentes por complicaciones en el puerperio. ${ }^{8}$ Siendo que estas complicaciones y el aborto figuran entre las cinco causas de muerte en mujeres de 15-19 años de edad. ${ }^{10}$

Ante lo expuesto, se reconoce que el distrito de San Juan de Lurigancho es el área con mayor población en Lima capital, alcanzando más de un millón de habitantes en el $2011(1004,339)$ y el $30,45 \%$ estaba comprendido por mujeres de 15-29 años. ${ }^{8,11} \mathrm{Al}$ respecto, Candela, basado en la información brindada por Almeyda, refiere que uno de cada cinco embarazos corresponde a adolescentes de 12-19 años, los cuales se producen principalmente en ese distrito. ${ }^{12}$

Actualmente, existen políticas de educación sexual que promueven una mayor sensibilización en el adolescente con participación de la familia, las instituciones escolares y la sociedad. Pero ¿Qué tan buena puede ser esta educación o que tan constante y permanente es? Es propio reconocer que la familia atraviesa un periodo difícil, sobre todo, porque ambos padres trabajan o conforman familias monoparentales. Aun así, la forma en cómo se concibe la sexualidad depende en gran medida de la influencia social, de cómo son los grupos de pertenencia en esta edad, comenzando por los padres o la familia, que marcan la idea de lo considerado normal en una persona del sexo opuesto.

En este contexto, la educación sexual es relevante, sobre todo, para promover comportamientos autónomos y ayudar a construir un sistema personal de control de la propia conducta (nivel psicológico) y enseñar un concepto más adecuado de libertad (nivel ético) al adolescente. ${ }^{13}$ Reconociendo que la mejor solución no es solo enseñar cuáles son los métodos anticonceptivos y cuál es más efectivo, sino 
fortalecer la educación en valores, reforzar la idea de una abstinencia saludable y orientar de forma real los beneficios y riesgos que implica iniciar una sexualidad precoz. ${ }^{14}$

La educación ética y la sexualidad son procesos paralelos e interconectados. Educar los deseos, impulsos, sentimientos y apegos, fortaleciendo la autonomía en el adolescente, en un plano de convivencia de valores, promoverá responsabilidad en la procreación y control del impulso; dado que la sexualidad se presenta como una colosal fuente de expectativas y de felicidad, experiencia que el adolescente desea disfrutar sin coacciones ni deberes, considerando la fidelidad como un valor caduco. ${ }^{15}$

Cordova $^{16}$ refiere que la educación debe fortalecer habilidades que promuevan en el adolescente autoconfianza y el respeto por sus pares, que le permita establecer adecuadas relaciones autónomas con ellos, de forma responsable y consciente. Para Minchón et al., ${ }^{17}$ los padres y profesores deben participar de la educación sexual, a fin de ofrecer una formación eficaz y eficiente; la cual debe formar parte del currículum educativo general.

Sin embargo, frente a una educación que se plasma como intencionada, integrada y formal, existe también la educación sexual paralela, aquella que ofrecen los medios masivos de comunicación y la cultura de la sociedad actual, quienes imponen que lo físico es más importante que lo interior; siendo la autoestima reemplazada por un traje o peinado de moda, fomentando en el adolescente decisiones riesgosas. En ese sentido, este estudio tuvo como objetivo determinar el nivel de conocimiento sobre educación sexual y describir el perfil sexual de una población de adolescentes de secundaria en el Distrito de San Juan de Lurigancho-Lima.

\section{METODOLOGÍA}

Estudio de enfoque cuantitativo y método observacional, de diseño descriptivo; realizado en dos instituciones educativas estatales localizadas en el distrito de San Juan de Lurigancho-Lima, ambos son centros con más de 1000 estudiantes, con nivel primario y secundario, siendo de carácter mixto y diurno.

La población fue constituida por estudiantes de $3^{\circ}, 4^{\circ}$ y $5^{\circ}$ año de secundaria, totalizando 1200 adolescentes con edades entre 14 y 17 años. La muestra fue determinada mediante formula de población finita, resultando en 449 estudiantes, quienes fueron seleccionados mediante muestreo aleatorio por conglomerado. La unidad de análisis fue el estudiante, participando 258 adolescentes de una institución y 191 de la segunda, según criterios de inclusión: ambos sexos, edad entre 14-17 años, asistencia regular a clase y participación voluntaria.

La técnica usada para la recolección de datos fue la encuesta y el instrumento un cuestionario estructurado, elaborado por la autora basado en la revisión de la literatura, que contenía 42 ítems, de los cuales 08 correspondían a los datos generales. El instrumento fue sometido a juicio de expertos y sus resultados se analizaron con la prueba binomial. En un segundo momento, el instrumento se sometió a prueba piloto con 50 adolescentes, quienes no formaron parte de la muestra final y cuyos resultados fueron analizados con el coeficiente Alfa de Cronbach para las respuestas politómicas del perfil sexual $(\mathrm{p}=0,77)$ y fuentes de información $(\mathrm{p}=0,66) \mathrm{y}$, mediante la prueba de $\mathrm{K}$ Richardson para las respuestas dicotómicas de sexualidad, ética y métodos anticonceptivos $(\mathrm{p}=0,89)$.

A cada respuesta fue asignado el valor de 1 a 4. Las preguntas del perfil sociodemográfico y sexual eran politómicas categorizadas de forma ordinal, considerándose un mejor perfil cuanto mayor era el puntaje; mientras que en conocimiento se consideraron respuestas dicotómicas con uso de distractores, calificándose con cero a la respuesta incorrecta y uno a la correcta.

Los datos se recolectaron de abril-junio del 2014, una vez cumplido los trámites administrativos en ambas instituciones; seguidamente se coordinó con los tutores de cada salón seleccionado y se invitó a los adolescentes a participar en el estudio, previa orientación y firma del consentimiento informado por los padres $\mathrm{y}$ asentimiento informado por ellos mismos.

La aplicación del cuestionario fue realizada en los respectivos salones en horario de tutoría, con un tiempo aproximado de 30 minutos. Culminada la fase de recolección, se desarrolló una sesión educativa sobre "Sexualidad y Educación Sexual" con los estudiantes, a modo de responsabilidad ética.

Previo al análisis de datos, se realizó el control de calidad verificando la información de 
cada cuestionario, actividad desarrollada por un tercero quien fue previamente capacitado. Luego, los datos fueron digitados en una base de datos en el Programa Excel 2010 utilizando códigos para cada participante, preservando así sus anonimatos. Finalmente, los datos fueron transportados al Programa SPSS versión 19.0 y procesados mediante la estadística descriptiva (porcentajes, frecuencias, medias, desviación estándar).

El Proyecto fue evaluado y aprobado por el Comité de Ética de la Facultad de Medicina de la Universidad Ricardo Palma (Oficio No. 0262014/CARD-AB) y Dirección de Salud - DISA IV Lima-Este (Oficio No. 67-2014-FG/OE-004-DISA IV LE). El estudio cumplió con los principios de Beneficencia, pues lo que buscaba en el Adolescente era conocer cuánto tiene internalizado conocimientos sobre educación sexual y cuál su perfil, a fin de reorientar acciones en su propio beneficio en la escuela y comunidad. Autonomía, porque los adolescentes participaron voluntariamente en el estudio, libre de presión o coacción. Justicia, porque todas/os la/os adolescentes fueron tratados de igual manera sin discriminación. No maleficencia, porque no se le causó daño físico y/o mental al adolescente.

\section{RESULTADOS}

Tabla 1: Datos generales de los adolescentes de secundaria, distrito de San Juan de Lurigancho. Abril-junio 2014.

\begin{tabular}{|c|c|c|c|}
\hline & Características & $\begin{array}{c}\text { Frecuencia } \\
(\mathrm{n}=449)\end{array}$ & $\begin{array}{c}\text { Porcentaje } \\
(100 \%)\end{array}$ \\
\hline \multirow{2}{*}{$\begin{array}{l}\stackrel{x}{x} \\
\stackrel{\leftrightarrow}{\infty}\end{array}$} & Femenino & 222 & 49 \\
\hline & Masculino & 227 & 51 \\
\hline \multirow{3}{*}{ 究 } & Tercer & 143 & 32 \\
\hline & Cuarto & 108 & 24 \\
\hline & Quinto & 198 & 44 \\
\hline \multirow{6}{*}{ 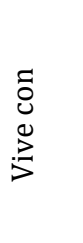 } & Ambos padres & 275 & 61 \\
\hline & Papá & 21 & 5 \\
\hline & Mamá & 119 & 27 \\
\hline & Hermanos & 11 & 2 \\
\hline & Abuelos & 3 & 1 \\
\hline & Otros & 20 & 4 \\
\hline \multirow{6}{*}{ 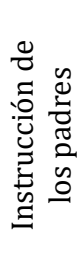 } & Universitario/técnico & 61 & 14 \\
\hline & Secundaria completa & 189 & 42 \\
\hline & Secundaria incompleta & 123 & 27 \\
\hline & Primaria completa & 36 & 8 \\
\hline & Primaria incompleta & 29 & 6 \\
\hline & Iletrado & 11 & 2 \\
\hline \multirow{4}{*}{ 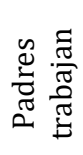 } & Ambos trabajan & 257 & 57 \\
\hline & Solo el papá & 89 & 20 \\
\hline & Solo la mamá & 48 & 11 \\
\hline & Ninguno & 55 & 12 \\
\hline
\end{tabular}

En la tabla 1, se observa que el $61 \%$ de adolescentes vivía con sus padres, quienes tenían secundaria completa $(42 \%)$ y en el $57 \%$ de casos ambos padres trabajaban. Asimismo, de los adolescentes encuestados el $44 \%$ pertenecía al $5^{\circ}$ grado de secundaria, con una edad promedio de 15,0 $\pm 1,03$ años de desviación estándar, siendo el $51 \%$ varones.

Gráfico 1: Perfil sexual de los adolescentes de secundaria, distrito de San Juan de Lurigancho. Abril-junio 2014.

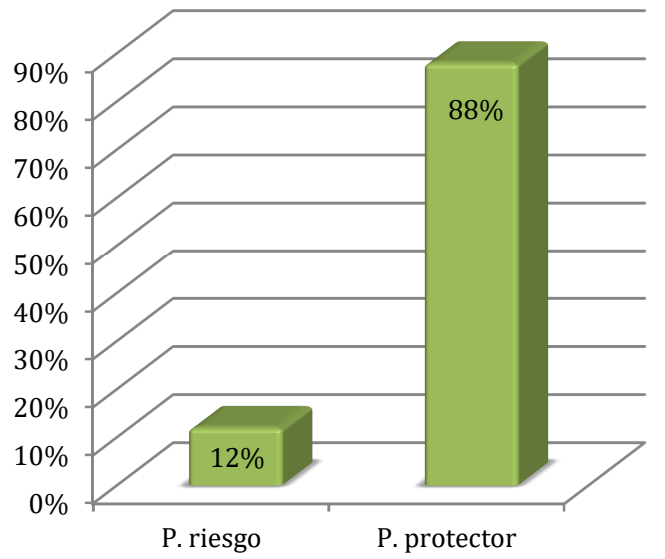

En el gráfico 1, se identifica que el 12\% (54) de estudiantes tenía un perfil sexual de riesgo versus el $88 \%$ (397) que tenía un perfil protector. En datos no mostrados, el 72,4\% (325) registró no haber tenido relaciones sexuales, de ellos $82,9 \%$ (184) era mujer; el $13,1 \%$ (60) lo hizo antes de los 14 años y entre ellos el 22,5\% (51) fue varón.

Gráfico 2: Perfil sexual según dimensiones de los adolescentes de secundaria, distrito de San Juan de Lurigancho. Abril-junio 2014.

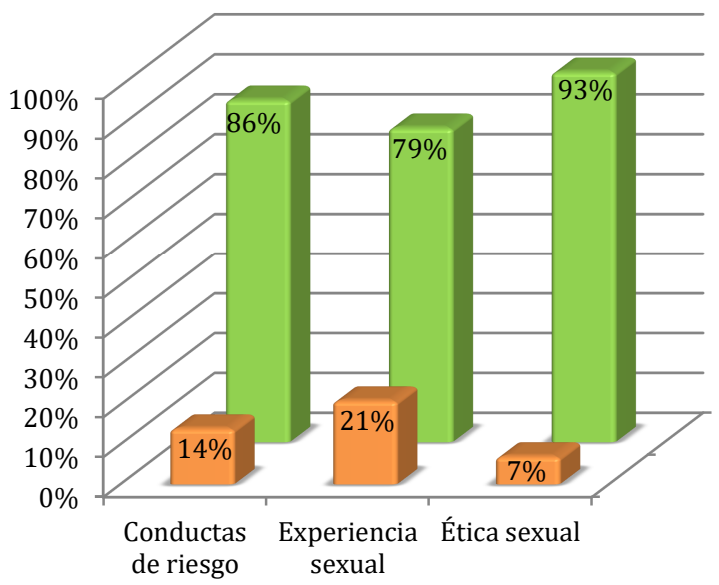

En el gráfico 2, sobre el análisis por dimensiones, se observa predominio de perfil 
protector en los adolescentes. Entre los datos no mostrados, en la dimensión "conductas sexuales de riesgo" el 13,4\% (60) de adolescentes registró haber tenido de cinco a más enamorados/as; $3,1 \%$ (14) se quedaba a solas con su enamorado/a, mientras que solo el 1,8\% (8) realizaba contacto de caricias en zonas íntimas con bastante frecuencia; otros 5,6\%(25) se masturbaban y 3,8\% (17) veía pornografía con bastante frecuencia.

En la dimensión "experiencia sexual", el $14,7 \%$ (66) de adolescentes afirmó haber tenido su primera experiencia sexual con un desconocido $\mathrm{y}$, solo el 1,6\% (7) tenía relaciones sexuales con bastante frecuencia al momento de la encuesta. Sobre el número de parejas sexuales, el 5,3\% (24) de adolescentes registró haber tenido más de tres parejas. En la dimensión "ética sexual”, el 63,9\% (287) consideró que los valores como el amor, el respeto, la libertad y la fidelidad en la vida sexual son muy importantes y el $3,1 \%$ (14) registró que dichos valores no lo son; siendo que el 37\% (166) siempre los practicaba y el $14,7 \%$ (66) nunca.

Gráfico 3: Conocimientos sobre educación sexual de los adolescentes de secundaria, distrito de San Juan de Lurigancho. Abril-junio 2014.

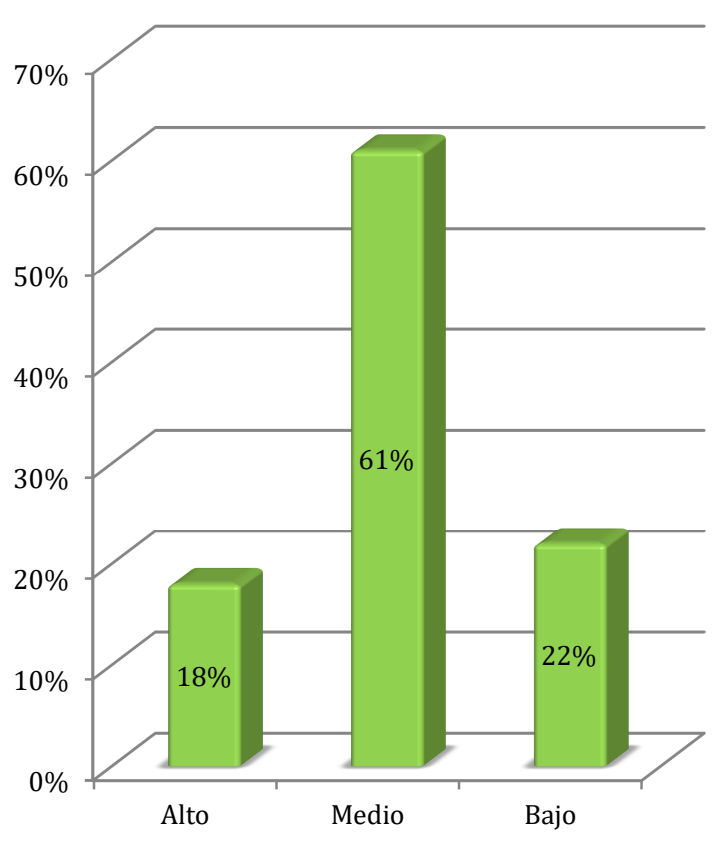

En el gráfico 3, se observa que el 61\% (273) de adolescentes presentó un nivel de conocimiento medio sobre educación sexual, mientras que solo el $18 \%$ (79) tenía un alto nivel de conocimiento, contenidos que se discriminan en el siguiente gráfico.
Gráfico 4: Conocimientos sobre educación sexual según dimensiones de los adolescentes de secundaria, distrito de San Juan de Lurigancho. Abril-junio 2014.

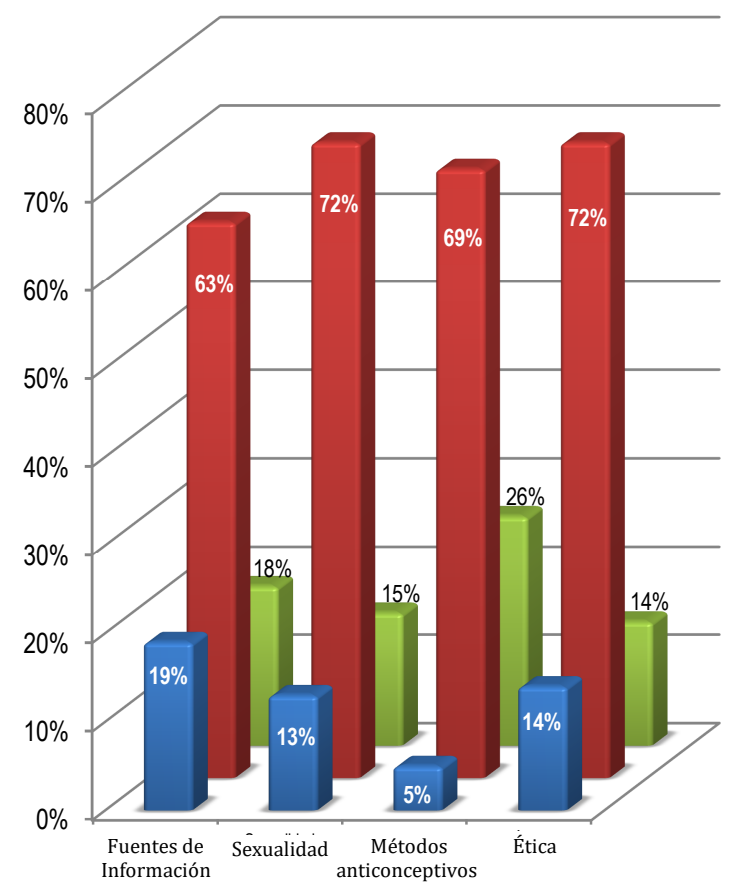

$\square$ Bajo $\square$ Medio $\square$ Alto

En el gráfico 4, sobre las dimensiones del conocimiento, existe predominio del nivel medio con valores semejantes. En la dimensión "Fuente de información", el 35,4\% (159) de adolescentes registró que algunas veces solía hablar sobre temas de sexualidad con sus padres; asimismo, el 47,9\% (215) expresó haber aprendido sobre el tema con su profesor en clase y $23,8 \%$ (107) refirió que sus dudas lo resolvía en internet. En "Sexualidad", el $85,1 \%$ (382) desconocía qué es la sexualidad y 64,1\% (288) educación sexual; asimismo, el 75,7\% (340) de estudiantes desconocía qué es la planificación familiar.

En la dimensión "Métodos anticonceptivos", el $85,1 \%$ (382) desconocía sobre los métodos existentes, 84,2 (378) sobre el uso correcto del preservativo y $79,7 \%$ (358) cuál es el método anticonceptivo más efectivo. Finalmente, en "Ética" que implica los valores y principios en la sexualidad, el $63,7 \% \quad(286)$ y $63,5 \% \quad$ (285) respectivamente, registró que desconocía cuáles se consideran los valores más importantes que deben preservarse en la amistad o relación de pareja o enamorado/a y, el 51,4\% (231) no sabía el significado del término "abstinencia" sexual. 
Tabla 2: Descripción de la primera experiencia de relación sexual según edad y sexo de los estudiantes del distrito de San Juan de Lurigancho. Abril-junio 2014.

\begin{tabular}{|c|c|c|c|c|c|c|c|c|c|c|c|}
\hline \multirow{3}{*}{ 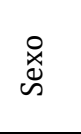 } & \multirow{3}{*}{ 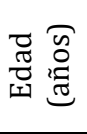 } & \multicolumn{8}{|c|}{ Edad de inicio de la vida sexual activa } & \multirow{2}{*}{\multicolumn{2}{|c|}{$\begin{array}{c}\text { TOTAL } \\
(100 \%)\end{array}$}} \\
\hline & & \multicolumn{2}{|c|}{$\leq 14$ años } & \multicolumn{2}{|c|}{$\geq 15$ años } & \multicolumn{2}{|c|}{$\geq 16$ años } & \multicolumn{2}{|c|}{ Inactivo } & & \\
\hline & & $*_{f}$ & $\%$ & $\mathrm{f}$ & $\%$ & $\mathrm{~F}$ & $\%$ & $\mathrm{~F}$ & $\%$ & $\mathrm{~F}$ & $\%$ \\
\hline \multirow{5}{*}{ 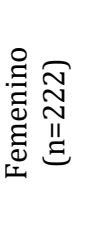 } & 13 & 2 & 0,90 & - & - & - & - & 16 & 7,20 & 18 & 8,11 \\
\hline & 14 & 4 & 1,80 & - & - & - & - & 41 & 18,47 & 45 & 20,27 \\
\hline & 15 & 1 & 0,45 & 13 & 5,86 & - & - & 81 & 36,48 & 95 & 42,79 \\
\hline & 16 & 3 & 1,35 & 8 & 3,60 & 6 & 2,70 & 37 & 16,67 & 54 & 24,32 \\
\hline & 17 & - & - & - & - & 1 & 0,45 & 9 & 4,05 & 10 & 4,50 \\
\hline \multirow{5}{*}{ 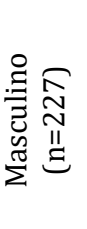 } & 13 & 4 & 1,76 & - & - & - & - & 11 & 4,85 & 15 & 6,61 \\
\hline & 14 & 15 & 6,61 & - & - & - & - & 37 & 16,29 & 52 & 22,90 \\
\hline & 15 & 16 & 7,05 & 7 & 3,08 & - & - & 54 & 23,79 & 77 & 33,92 \\
\hline & 16 & 11 & 4,85 & 6 & 2,64 & 8 & 3,52 & 34 & 15,00 & 59 & 26,00 \\
\hline & 17 & 5 & 2,20 & 7 & 3,08 & 7 & 3,08 & 5 & 2,20 & 24 & 10,56 \\
\hline
\end{tabular}

$* \mathrm{f}=$ frecuencia.

En la tabla 2, de los 449 adolescentes encuestados; en el grupo de mujeres $(n=222)$ se identifica que el 4,5\% (10) tuvo su primera experiencia sexual antes de los 14 años y 9,45\% (21) a los 15 años, sumando ambas edades esta cifra alcanza el 13,96\%; destacando que el 82,88\% (184) aun no tenía esa experiencia. En el grupo de varones, el 22,46\% (51) lo había tenido antes de los 14 años y $8,81 \%$ (20) antes de los 15; ambas edades alcanzan el $31,27 \%$ (71). En general $(n=449)$, el $13,58 \%$ (61) de la población encuestada había tenido su primera experiencia sexual antes de los 14 o, a los 14 años y, el 9,13\% (41) antes de los 15 o, a los 15 años, sumando estas edades este porcentaje en la población alcanza al 22,79\%. Identificándose que el $82,88 \%$ (184) de mujeres versus el $62,11 \%$ (141) de varones aún era casto.

\section{DISCUSIÓN}

Actualmente, el adolescente enfrenta múltiples factores de riesgo que le provocan enfermedades o situaciones que impiden su adecuado crecimiento y desarrollo, tanto a nivel fisiológico y psicológico, como social y económico. Ante éstos, también debe hacer frente a su sexualidad y buen uso, siendo que en el descubrimiento y facetas de cambios de ésta, el adolescente se expone a consecuencias que pueden desencadenarle frustraciones, como el embarazo precoz, las ITS y el VIH-Sida.

En el estudio, el $51 \%$ de adolescentes era de sexo masculino, con una media de edad de 15 años (tabla 1), a diferencia de estudio previo. ${ }^{18}$ Edad que corresponde a la etapa tardía de la adolescencia según la OMS, ${ }^{1}$ en la que, a pesar de existir una mayor independencia respecto a los padres, aun es necesario contar con un guía permanente; en ese sentido, contar con una familia nuclear puede ser un factor protector en los adolescentes, especialmente cuando existe una buena comunicación familiar, tal es así que el 35,4\% habla con sus padres sobre temas sexuales algunas veces (gráfico 4).

En la infancia, la comunicación es fluida, permanente, directa y sin vergüenza en contexto familiar, dado que el niño ve al padre como su mejor amigo y único guía; mas, en la adolescencia, caracterizada como una época de rebeldía, ante la búsqueda de autonomía y deseo permanente de tener la razón, a pesar de errar, dicha comunicación se anula; lo que podría evitarse si los padres fundamentan su relación familiar en valores, comprensión, armonía y confianza, asegurando una buena y permanente comunicación. ${ }^{19}$

Aún hoy, que muchos padres aprueban la enseñanza de la educación sexual en las instituciones escolares, en el hogar es tema de rechazo, en su mayoría por razones religiosas y/o morales, sin darse cuenta que hablar de sexualidad no solo es referirse al impulso sexual dirigido al placer y la reproducción, sino que también aborda aspectos psicológicos y espirituales en el desarrollo físico-emocional del adolescente. ${ }^{20} \mathrm{De}$ continuarse negando la educación sexual en el hogar, sea por censura, falta de conocimiento o temor de los padres, los hijos tenderán a presentar mayor duda y consecuentemente mayor predisposición para un pronto inicio de su actividad sexual. ${ }^{21}$ Proceso que debe ser acompañado y monitorizado por los padres $\mathrm{y}$ 
personas significativas, para evitar consecuencias indeseables para la salud y la vida.

El estudio de Gonzáles, ${ }^{22}$ muestra que el $59 \%$ de adolescentes vivía dentro de una familia nuclear; pero entre aquellos que tenían una vida sexual activa (17\%), el $8 \%$ procedía de familias con disfunción severa. Resaltando que el inicio precoz estaría relacionado con un bloqueo en la comunicación en las familias.

En relación al perfil sexual del adolecente, el $12 \%$ de adolescentes en el estudio tenía un perfil sexual de riesgo, bastante menos a lo observado en previos estudios, como el de Trejo-Ortiz et al. ${ }^{18} \mathrm{y}$ Parra-Villarroel y Pérez-Villegas. ${ }^{23}$ Identificándose como positivo este cambio, ya que luego de tres años este porcentaje se redujo en más de $15 \%$, lo que puede ser reflejo del impacto de las estrategias educativas que se vienen desarrollando en las denominadas Escuelas Saludables, donde la educación sexual es parte del currículo; siendo que los estudiantes en situación de embarazo y paternidad precoz son tratados con especial consideración y acompañados de cerca por sus tutores. Realidad que puede inducir a reflexionar a los compañeros de clase y tomar mejores decisiones para sus vidas.

Datos de la ENDES $2013^{24}$ muestra que el embarazo adolescente continua en aumento (de $13,2 \%$ en el 2012 pasó a 13,9\% en el 2013) y SJL ocupa el primer lugar. Mientras tanto, en el estudio se identificó que el 72,4\% (325) de adolescentes aún era casto al momento de la encuesta (tabla 2). $\mathrm{Al}$ respecto, se puede conjeturar dos posturas: 1) es posible que los estudiantes hayan ocultados la verdad por pudor o, 2) que realmente el hecho de formar parte de una familia nuclear $(61 \%)$ sea un factor protector para retrasar el inicio de la vida sexual (tabla 1), hecho que estaría reforzado por las formas de abordar la sexualidad en las instituciones participantes en el estudio.

Actualmente, las instituciones escolares informan al adolescente sobre sexualidad enseñándoles sobre los métodos anticonceptivos. Sin embargo, sería importante también que se introduzcan temas de valores y su aplicación en la vida diaria del adolescente, promoviéndose el fortalecimiento de su personalidad y autoestima. ${ }^{14,16}$ En términos claros, debería hablarse sobre la planificación familiar, explicando que no solo es liberación del impulso sexual, sino que es el conocimiento de la responsabilidad que implica y la toma de afecto $^{7}$ en la relación, que involucra sentimientos de respeto, de igualdad y de protección del otro/a.

En el estudio, el $61 \%$ de adolescentes presentó un conocimiento medio (gráfico 3), datos contrarios al de Salazar, ${ }^{25}$ dado que el $65 \%$ de su población tuvo un bajo nivel de conocimiento sobre educación sexual, específicamente en métodos anticonceptivos; semejantes al estudio de Rojas. ${ }^{26}$ Hasta el 2010, época en que se realizaron ambos estudios, el currículo no incluía la educación sexual, pues era visto como tema inapropiado para las conversaciones en clase. Los propios docentes sabían muy poco sobre ello y unos pocos habían participado de algún curso de capacitación, ${ }^{26}$ lo que corrobora el hecho de que en ese entonces el tema era tratado con restricción, realidad que aún puede estar ocurriendo, pues existe la idea que hablar de sexualidad y educación es promover la vida sexual activa, percepción errónea al observar el incremento del embarazo precoz actualmente.

Llama la atención en el estudio, los resultados del gráfico 4, que discrimina las respuestas de los estudiantes por dimensiones, siendo que en "Sexualidad" desconocían el significado del propio término y, más aun, qué es educación sexual y planificación familiar; asimismo, la mayoría desconocía sobre los métodos anticonceptivos, en quienes los valores de respeto, fidelidad y libertad tenían poca importancia. Datos que evidencian un vacío cognitivo para una práctica saludable de la vida sexual, no apenas consumando el coito, sino más bien, como fundamento de un modo de vivir, basado en la abstinencia y la madurez del proceso sexual responsable y ético.

Otro dato relevante, es que el 65,7\% (295) de adolescentes consideró que las decisiones u opiniones de sus enamorados/as no son importantes, siendo que $57 \%$ (248) registró que la virginidad y/o castidad tampoco lo es. Información que devela el pensamiento del adolescente postmoderno, lejos de los valores de castidad y obediencia; aspecto que debe ser revalorizado dentro del contexto familiar, no solo con argumentos sino con la práctica, con la forma de vida de los padres y personas representativas para el adolescente, siendo que aún están en fase de búsqueda de figuras que influirían fuertemente en la definición de su carácter y personalidad.

Frente a estos datos, a pesar de las múltiples estrategias implementadas en la educación sexual 
para el adolescente, aún se viene desconsiderando un aspecto relevante en el ejercicio de la vida sexual, que es la ética; tema de poco realce en la sociedad actual, en la que proliferan desnudos, cosificación de la mujer y el dominio del varón sobre la mujer; hechos que se pueden evidenciar a diario en los diversos medios y programas de comunicación masiva que, en vez de educar, distorsionan la relación entre el varón y la mujer.

Actualmente, el MINSA ${ }^{27}$ instaló la Mesa Intersectorial para la Prevención del Embarazo Adolescente en la Red de Salud de SJL, a fin de controlar la maternidad temprana en el distrito, considerando que representan a las futuras generaciones de progreso social, económico y político en el país. Esta acción tiene como propósito postergar el inicio de las relaciones sexuales en esta población, promoviendo una mayor escolarización en los adolescentes, dado el alto índice de abandono escolar. ${ }^{28}$

La acción, a través de un plan, pretende garantizar la inclusión de la educación sexual en el currículo e incrementar el uso de métodos anticonceptivos entre los adolescentes sexualmente activos; aunque también debería considerar temas sobre valores y principios éticos, además de aclarar que no existe mejor protección y seguridad que la abstinencia.

En esa perspectiva, es importante trabajar colaborativamente con el personal de salud, fomentando la creación de escenarios de promoción de la salud sexual y reproductiva basada en valores. Para tal, la enfermera se constituye en recurso humano elemental, por su permanente labor en la comunidad y su función educadora, debiendo promover cambios en la actitud cognitiva y luego comportamental, ya que

\section{Correspondencia:}

Alejandra Francesca Parodi Carro

Correo electrónico: alejandra.parodic@ @otmail.com

\section{REFERENCIAS BIBLIOGRÁFICAS}

1. Vega MG. Programa Conjunto de las Naciones Unidas sobre el VIH/Sida y Fondo de Población de las Naciones Unidas [internet]. 2012 [citado 18 de ago 2012]. Disponible en: http://www.un.org/spanish/sg/mop/gwa.shtml\# UNAIDS.

2. CDC: Centers for Disease Control and Prevention. Reproductive Health: Teens el conocimiento es la base para cuidarse a sí mismo, como lo refiere Orem, en su teoría que incentiva la práctica de actividades que realizan los individuos en su propio beneficio, manteniendo una buena calidad de vida, salud y bienestar.

La enfermera en este sentido, participa y promueve el buen crecimiento y maduración del adolescente, ayudándole a suplir las carencias de conocimiento para el desarrollo de su totalidad, incluyendo necesidades físicas, psicológicas y espirituales, actividad en la cual debe involucrar a los padres de familia, a fin de contar con sus apoyo en los momentos en que el profesional esté ausente. Especialmente porque son los padres los llamados a brindar el apoyo directo a los adolescentes, en los momentos de ambigüedad, duda, miedos y ansiedades.

Dichas actividades ayudarían a controlar y postergar el inicio de una vida sexual activa en el adolescente, disminuyendo a largo plazo las tasas de embarazo precoz, ITS y potenciales casos de VIH-Sida en el distrito de SJL, resultados que redundarían en bienestar de los propios adolescentes y del país como un todo.

A modo de conclusión, se destaca que el 51\% de adolescentes del estudio era de sexo masculino y $61 \%$ contaba con familia nuclear. Menos del $50 \%$ de los padres tenía estudios de nivel secundario y $57 \%$ trabajaba. Respecto al perfil sexual, el $12 \%$ de adolescentes era activo (perfil de riesgo); siendo que la edad de inicio sexual fue antes de los 14 años, en su mayoría varones. En cuanto al conocimiento sobre educación sexual, el $61 \%$ alcanzó un nivel medio y $22 \%$ bajo; habiendo menor conocimiento en los temas de métodos anticonceptivos y ética sobre educación sexual (mayor a 60\%). pregnacy [homepage de internet]. Atlanta USA: CDC; 2012 [actualizado 13 mar 2013; citado 20 de nov 2013]. Disponible en: http://www.cdc.gov/TeenPregnancy/About TeenPreg.htm.

3. UNICEF: Fondo de las Naciones Unidas para la Infancia. Estado Mundial de la Infancia 2011. La adolescencia. Una época de 
oportunidades [internet]. New York: UNICEF; Feb 2011 [citado 15 de ago 2012]. 140 p. Disponible en: http://www.unicef.org/devpro/files/SOWC_201 1_Main_Report_SP 02092011.pdf

4. Bárcena A. Embarazo adolescente, alerta temprana de la desigualdad [homepage de internet]. Washitong DC: Comisión Económica para América Latina; Jul 2013 [actualizado 08 de jul 2013; citado 10 de mar 2015]. Disponible en: http://www.cepal.org/es/articulos/embarazoadolescente-alerta-temprana-de-la-desigualdad

5. UNICEF: Fondo de las Naciones Unidas para la Infancia. La Infancia y el SIDA: Quinto inventario de la situación, 2010 [internet]. New York: UNICEF; Nov 2010 [citado 10 de nov 2012]. 50 p. Disponible en: http://www.unicef.org/spanish/publications/file s/Children_and_AIDS-

Fifth_Stocktaking_Report_2010_SP.pdf.

6. MINSA: Ministerio de Salud-Perú. Salud de las y los adolescentes Peruanos. Ubicándolos y Ubicándonos [internet]. Lima: MINSA; 2012. [citado 10 de set 2012]. 10 p. Disponible en: http://www.minsa.gob.pe/portada/Especiales/2 009/adolescentes/archivos/boletin02.pdf

7. Acevedo L. Embarazo en adolescentes [homepage de internet]. UNICEF. República Dominicana; 2012 [actualizado SN; citado 15 de oct 2012]. Disponible en: http://www.unicef.org/republicadominicana/he alth childhood 10191.htm

8. INEI: Instituto Nacional de Estadística e Informática. Encuesta Demográfica y de Salud Familiar 2012 [internet]. Lima: INEI; 2012 [citado 20 de set 2012] 438 p. Disponible en: http://proyectos.inei.gob.pe/endes/2012/.

9. INPPARES: Instituto Peruano de Paternidad Responsable. Políticas Públicas Acción de Institucionalidad. Art. 173-3. Código Penal. Derechos Vulnerados. Planteamiento, avances y siguientes pasos [internet]. Lima: INPPARES; 2010. [citado 18 de set 2012]. 12p. Disponible en: http://www.inppares.org/sites/default/files/Cam paFirmasparawebMayo.pdf

10. INEI: Instituto Nacional de Estadística e Informática. Perú: Situación Social de las madres adolescentes 2007 [internet]. Lima: INEI; Mar 2007 [citado 22 de nov 2012]. 132 p. Disponible en: http://www.unfpa.org.pe/publicaciones/publica cionesperu/INEI-Peru-Situacion-social-madresadolescentes.pdf

11. Veliz SMV, Uribe PAW, Fuertes MDP, Aquino YNG, Valdivia VEP, Benites AYL. Análisis de la situación de salud de los servicios hospitalarios ASISHO-2012 [internet]. San Juan de Lurigancho-Lima: Hospital San Juan de Lurigancho. Unidad de Epidemiología y Salud Mental; May 2012 [citado 15 de dic 2012]. 169 p. Disponible en: http://www.hospitalsjl.gob.pe/ArchivosDescarg a/Epidemiologia/ASIS/ASISHO2012.pdf.

12. Candela FL. San Juan de Lurigancho Presenta más casos de adolescentes embarazadas [homepage de internet]. San Juan de Lurigancho-Lima: San Juan de Lurigancho; Oct 2005 [actualizado SN; citado 15 de dic 2012]. Disponible en: http://www.sjl.pe/noticias/noticia.asp?id=1021

13. OMS: Organización Mundial de la Salud. Temas de salud: Salud de los Adolescentes [homepage de internet]. Ginebra: OMS; 2013 [actualizado SN; citado 10 de mar 2013]. Disponible en: http://www.who.int/topics/adolescent_health/es/

14. Caricote AE. Los valores en la educación sexual del adolescente. Revista de Educación en Valores [serie de internet]. Jul-dic 2010 [citado 10 de gos 2013]. 2 (14): 76-88. Disponible en: http://servicio.bc.uc.edu.ve/multidisciplinarias/ educacion-en-valores/v1n14/art9.pdf

15. Marina JA. La Sexualidad y la ética. Estudios de Juventud [serie de internet]. 2003 [citado 15 de ago 2013]; 63: 9-17. Disponible en: http://www.injuve.es/sites/default/files/03\%20 SEXUALIDAD\%20Y\%20ETICA.pdf

16. Córdova J. Valores, Normas, Actitudes y Comportamientos en Adolescentes y Jóvenes [internet]. Paraguay: Alianza para la Salud; Jun 2004 [citado 10 de jul 2013]. 75 p. Disponible en: http://www.cird.org.py/salud/docs/Informe_Ali anza_IEC.pdf 
17. Minchón CM, Oliva DMA, Domínguez PS. Intervención Enfermera ante los Embarazos no deseados. Enfermería Global [serie de internet]. Feb 2008 [citado: 15 de abr 2013]; 12: [aproximadamente 09 laudas]. Disponible en: https://digitum.um.es/xmlui/bitstream/10201/2 4269/1/Intervencion20enfermera20ante20los20 embarazos20no20deseados..pdf

18. Trejo-Ortíz PM, Moreno-Chávez PC, MacíasAguilar M, Valdez-Esparza G, Mollinedo MFE, Lugo BLG, et al. Conocimiento y comportamiento sexual en adolescentes. Área Académica Ciencias de la Salud. Universidad Autónoma de Zacatecas. Rev Cubana Enfermer [serie de internet]. Set-dic 2011 [citado: 15 de ago 2012]; 27(4): 273-80. Disponible en: http://scielo.sld.cu/pdf/enf/v27n4/enf05411.pdf

19. Ibarra HKS, Meneses RS. Sexualidad y Comunicación en la familia. Estudio de caso en alumnos del instituto de ciencias sociales y humanidades. Área académica de ciencias de la comunicación [internet]. México: Universidad Autónoma del Estado de Hidalgo; 2012 [citado: 10 de jun 2013]. 85 p. Disponible en: http://www.uaeh.edu.mx/docencia/Tesis/icshu/l icenciatura/documentos/Sexualidadycomunicac ion.pdf.

20. Domínguez DI. Influencia de la familia en la sexualidad adolescente. Rev Cubana Obstet Ginecol [serie de internet]. Jul-sep 2011 [citado: 15 de jul 2013]; 37(3): 387-98. Disponible

en: http://scielo.sld.cu/pdf/gin/v37n3/gin11311.pdf

21. Salazar-Granara A, Maria-Álvarez AS, SolanoRomero I, Lázaro-Vivas Katherine, ArrolloSolis S, Araujo-Tocas V et al. Conocimientos de sexualidad, inicio de relaciones sexuales y comunicación familiar, en adolescentes de Instituciones Educativas nacionales del distrito del Agustino, Lima-Perú. Horiz Med [serie de internet]. Dic 2007 [citado: 10 de jul 2013]; 7(2): 79-85. Disponible en: http://www.medicina.usmp.edu.pe/horizonte/20 07_II/Art3_Vol7_N2.pdf.

22. González JC. Conocimientos, Actitudes y Prácticas sobre la Sexualidad en una Población Adolescente Escolar. Rev Salud Pública [serie de internet]. Feb 2009 [citado: 10 de ago 2012]; 11(1):14-26. Disponible en: http://www.redalyc.org/articulo.oa?id=422111 $\underline{03}$
23. Parra-Villarroel J, Pérez-Villegas R. Comportamiento sexual en adolescentes y su relación con variables biosociales. Perinatol Reprod Hum [serie de internet]. Ene-mar 2010 [citado 10 de ago 2012]; 24(1): 7-19. Disponible en: http://www.medigraphic.com/pdfs/inper/ip2010/ip101b.pdf.

24. INEI; Instituto Nacional de Estadística e Informática. Encuesta Demográfica y de Salud Familiar-ENDES 2013 [internet]. Lima-Perú: INEI; 2013 [citado 12 de oct 2014]. 478 p. Disponible en: http://www.inei.gob.pe/media/MenuRecursivo/p ublicaciones digitales/Est/Lib1151/index.html

25. Salazar JH. Conductas, Conocimiento y Actitudes de Estudiantes Adolescentes de zonas urbanas de las ciudades de Cusco e Iquitos frente a los Métodos Anticonceptivos. Rev. Psicol Herediana [serie de internet]. 2007 [citado 10 de ago 2012]; 2(1): $34 . \quad$ Disponible en: http://www.upch.edu.pe/fapsi/rph/NUMERO/c onductaspdf.

26. Rojas SJM. Nivel de Conocimientos y Práctica Enseñanza-Aprendizaje sobre Educación Sexual en Profesores y Estudiantes de 4to y 5to de secundaria de la I.E. $\mathrm{n}^{\circ} 1174$ "Virgen del Carmen" [tesis de licenciatura]. Lima-Perú: Universidad Ricardo Palma; 2010.

27. MINSA: Ministerio de Salud-Perú. Mesa Intersectorial para prevenir el embarazo en adolescentes en SJL 2014 [internet]. Lima: MINSA; Jun 2014 [citado 10 de oct 2014]. [aproximadamente 05 laudas]. Disponible en: http://www.minsa.gob.pe/portada/prensa/mas_ $\underline{\text { notas.asp? } \text { nota }=14540}$

28. MINSA: Ministerio de Salud-Perú. Plan Operativo Anual 2014. Hospital San Juan de Lurigancho [internet]. San Juan de LuriganchoLima; MINSA; Dic 2013 [citado: 15 de oct 2014]. 127 p. Disponible en: http://www.hospitalsjl.gob.pe/ArchivosDescarg a/Transparencia/PlaneamientoEstrategico/Plan OperativoInstitucional2014.pdf 